\title{
Vingt-cinq ans de publication périodique en bibliothéconomie au Québec : analyse quantitative du Bulletin de l'ACBLF / \\ Documentation et bibliothèques (1955-1979) \\ Twenty-five years of periodical publications about library \\ science in Quebec: a quantitative analysis of Bulletin de \\ l'ACBLF / Documentation et bibliothèques (1955-1979) \\ Veinticinco años de publicación periódica en biblioteconomia en Quebec: análisis cuantitativo de Bulletin de l'ACBLF / Documentation et bibliothèques (1955-1979)
}

Marcel Lajeunesse et Lise Wilson

Volume 27, numéro 2, juin 1981

URI : https://id.erudit.org/iderudit/1053817ar

DOI : https://doi.org/10.7202/1053817ar

Aller au sommaire du numéro

Éditeur(s)

Association pour l'avancement des sciences et des techniques de la

documentation (ASTED)

ISSN

0315-2340 (imprimé)

2291-8949 (numérique)

Découvrir la revue

Citer cet article

Lajeunesse, M. \& Wilson, L. (1981). Vingt-cinq ans de publication périodique en bibliothéconomie au Québec : analyse quantitative du Bulletin de l'ACBLF / Documentation et bibliothèques (1955-1979). Documentation et bibliothèques, 27(2), 53-67. https://doi.org/10.7202/1053817ar
Résumé de l'article

L'analyse de vingt-cinq années de publication du Bulletin de l'ACBLF/Documentation et bibliothèques (1955-1979) amène les auteurs à présenter les principales caractéristiques des collaborateurs qui ont contribué à la revue au cours de cette période et à décrire les articles, les comptes rendus et leur contenu. Ils tracent les grands traits de l'évolution de ce périodique et parallèlement de la profession et de la discipline documentaire au Québec.
Tous droits réservés (C) Association pour l'avancement des sciences et des techniques de la documentation (ASTED), 1981
Ce document est protégé par la loi sur le droit d'auteur. L'utilisation des services d'Érudit (y compris la reproduction) est assujettie à sa politique d'utilisation que vous pouvez consulter en ligne.

https://apropos.erudit.org/fr/usagers/politique-dutilisation/ 


\title{
Vingt-cinq ans de publication périodique en bibliothéconomie au Québec: analyse quantitative du Bulletin de l'ACBLF/Documentation et bibliothèques (1955-1979)
}

\author{
Marcel Lajeunesse \\ Université de Montréal \\ Lise Wilson \\ Collège de Maisonneuve \\ Montréal
}

L'analyse de vingt-cinq années de publication du Bulletin de l'ACBLF/Documentation et bibliothèques (1955-1979) amène les auteurs à présenter les principales caractéristiques des collaborateurs qui ont contribué à la revue au cours de cette période et à décrire les articles, les comptes rendus et leur contenu. Ils tracent les grands traits de l'évolution de ce périodique et parallèlement de la profession et de la discipline documentaire au Québec.

Twenty-five years of periodical publications about library science in Quebec : a quantitative analysis of Bulletin de l'ACBLF/Documentation et bibliothèques (1955-1979)

Through the analysis of the publication of Bulletin de l'ACBLF/Documentation et bibliothèques (19551979) over the last twenty-five years, the authors present the main characteristics of the contributors during this period and describe the articles, the summaries and their contents. In parallel, they outline the major development stages of the periodical and of documentation as a profession and a discipline in Quebec.

Velnticinco años de publicaclón periódica en blblloteconomia en Quebec: anállsis cuantitativo de Bulletin de l'ACBLF/Documentation et bibliothèques (1955-1979)

El análisis de veinticinco años de publicación de Bulletin de l'ACBLF/Documentation et bibliothèques (1955-1979) lleva los autores a presentar las principales características de los colaboradores que contribuyeron a la revista en este periodo y a describir los artículos, los resúmenes y su contenido. Destacan las caracteristicas de la evolución de la revista $y$, en paralelo, de la documentación como profesión y disciplina en Quebec.

Un périodique est un pourvoyeur essentiel d'information pour la communauté scientifique et professionnelle. Ainsi il n'est pas surprenant que l'une des premières actions d'une institution, d'une association volontaire, d'un groupe professionnel soit de se donner un bulletin ou une revue, véhicule d'information et de recherche qui rassemble ses membres et concourt à l'avancement d'une discipline.

Deux ans après sa fondation, l'Association canadienne des bibliothécaires catholiques lançait, en 1945, la Revue des bibliothèques ${ }^{1}$. En 1947, cette revue, la première en bibliothéconomie publiée en langue française en Amérique du Nord, cessait de paraître. De 1947 à 1954, une section de la revue Lectures intitulée "Bibliotheca " servait de publica-

1. J.-M. Bernard Léveillé, "Les bibliothécaires canadiensfrançais et leurs trente-deux ans de vie en association (ACBI - ACBLF - ASTED)", in G.-A. Chartrand, Livre, bibliothèque et culture québécoise, Montréal, ASTED, 1977, p. 708. tion officielle à l'Association². En mars 1955, l'Association canadienne des bibliothécaires de langue française lançait la première livraison de son Bulletin officiel; la présidente de l'Association, Juliette Chabot, y exprimait le vœu que ce Bulletin soit le moyen d'un "échange réel qui suscite des problèmes variés, ouvre des perspectives nouvelles et provoque une véritable communication des idées importantes pour l'avenir de notre profession »3.

Du Bulletin de mars 1955 à Documentation et bibliothèques de décembre 1979, beaucoup de changements ont été apportés par les divers comités de rédaction. Ce n'est pas notre propos de faire ici un historique détaillé de cette évolution, mais plutôt de souligner des étapes essentielles qui

2. Georges-A. Chartrand, "L'odyssée de notre publication officielle", Bulletin de l'ACBLF, vol. 9, no 2 (juin 1963), 102103.

3. Juliette Chabot, "Présentation ", Bulletin de l'ACBLF, vol. 1, no 1 (mars 1955), 3. 
rejoignent les objectifs de la présente étude. A partir de décembre 1961, la "chronique bibliographique» n'accepte pour recension que les ouvrages concernant la bibliothéconomie ou une science connexe ${ }^{4}$. En 1963, en même temps qu'il modernisait sa présentation graphique, le Bulletin de l'ACBLF sentait le besoin d'une politique rédactionnelle mieux définie et se voulait une revue réellement spécialisée 5 . Mais ceci ne pouvait se réaliser qu'en larguant une portion du contenu du bulletin. En 1965, avec la parution des Nouvelles de l'ACBLF, le Bulletin de l'ACBLF pouvait viser à présenter des articles originaux, qui résultaient “d'une pensée, d'une réflexion»6. En mars 1973, le Bulletin de l'ACBLF change son nom en celui de Documentation et bibliothèques. Celui-ci se prétendait «moins le véhicule de l'Association qu'une publication qui veut refléter les problèmes du milieu et les divers courants d'idées qui circulent parmi les membres ${ }^{7}$; elle voulait aussi définir et appliquer une acception plus large de la bibliothéconomie et poursuivre l'objectif de l'établissement d'une revue québécoise de recherche en information documentaire au Québec.

Une revue est un ensemble de messages. Dans une période de moyenne durée (25 ans), la revue que nous étudions doit refléter l'histoire intellectuelle de la profession et de la discipline documentaire au Québec, d'autant plus que cette revue a été, pour la plus grande portion de cette période, la seule revue de bibliothéconomie au Québec. En cela, nous acceptons l'énoncé du professeur Leon Carnovsky qui notait que

library periodicals are of necessity tailored to the demands of the members of the library profession and are, in turn, subject to the kinds of scholarly activity in which they engage, and which they write about 8 .

En réalité, quand nous étudions notre documentation périodique, c'est-à-dire notre pensée pendant un temps défini, c'est nous-mêmes que nous étudions. Cela a été démontré par d'éclairantes études tant dans notre discipline ${ }^{9}$ que dans d'autres ${ }^{10}$.

4. Bulletin de l'ACBLF, vol. 7 , no 4 (décembre 1961), 144

5. Paul Francœur, "Liminaire ", Bulletin de l'ACBLF, vol.9, no 1 (mars 1963), 2.

6. Yves Ducharme, "Liminaire", Bulletin de l'ACBLF, vol. 11, no 2 (juin 1965), 50.

7. "Mot de la rédaction", Documentation et bibliothèques, vol 19, no 1 (mars 1973), 2.

8. Leon Carnovsky, "Standards for library periodicals", Library Journal 80 (February 1955), 266

9. Voir notamment Virginia L. Allanson, Profile of a Library Magazine; Fifty Years of the Wilson Library Bulletin, Kent State University, School of Library Science, 1970, 59 p.; Mary Bloomfield, "The writing habits of librarians", College and Research Libraries, vol.27, no. 2 (March 1966), 109-119; Paula De Simone Watson, "Publication activity among academic librarians», College and Research Libraries, vol. 38, no. 5 (September 1977), 375-384 ; John N. Olsgaard and Jane Kinch Olsgaard, "Authorship in five library periodicals", College and Research Libraries, vol. 41, no. 1 (January 1980), 49-53; Donald J. Lehnus, «JEL, 1960-1970; an analytical study", Journal of Education for Librarianship, vol. 12, no. 2 (Fall 1971), 71-83.
Tout en nous inspirant de cette technique de recherche pour la description objective, systématique et quantitative du contenu manifeste des communications qu'est l'analyse du contenu'11, nous avons gardé de celle-ci la fonction heuristique pour expliquer l'évolution du périodique. Nous posons de nombreuses questions aux auteurs, nous étudions les sujets des articles ou des volumes analysés, le genre d'articles et les références infrapaginales. Dans cette étude qui se veut quantitative, mais avec des incursions au plan qualitatif, nous pensons déceler des tendances et des traits caractéristiques de l'évolution de la revue.

\section{Méthodologie}

II convient de rappeler, avant d'exposer la méthodologie que nous avons empruntée, l'hypothèse de travail qui nous a guidés tout au long de cette analyse quantitative. Nous avons voulu, par cette recherche, mettre en évidence les transformations qu'a connues le Bulletin de l'ACBFL / Documentation et bibliothèques et par conséquent saisir l'évolution qui a marqué la bibliothéconomie québécoise au cours des années 1955-1979.

L'étude s'est donc limitée aux écrits de cette période de vingt-cinq ans. Nous avons distingué deux types d'objets d'analyse, soit les articles proprement dits et les comptes rendus. Nous entendons par "article», toute contribution comptant plus d'un quart de page rédigée par un ou plusieurs auteurs connus ou anonymes. De la sorte, tous les articles ont été retenus, qu'il s'agisse des liminaires, des éditoriaux ou autres à l'exception cependant des annonces, des encarts publicitaires, des nouvelles brèves et de la chronique "Revue de presse". L'autre type de contribution que nous avons choisi d'examiner est le "compte rendu". Sont inclus dans cette catégorie tous les écrits dont la substance est l'analyse de monographies, de périodiques ou autres.

Au départ, nous n'avions pas la prétention de juger la qualité des articles présentés dans la revue. Nous avons donc privilégié une approche essentiellement quantitative en nous réservant toutefois la possibilité de juger qualitativement le contenu des articles dans le but d'établir leur typologie et de déterminer la méthode employée par les auteurs. Nous reviendrons sur cette question lorsque nous présenterons les variables retenues pour l'analyse.

Avant d'entreprendre l'étude en tant que telle, nous devions nous doter de deux grilles d'analyse afin de nous assurer de l'uniformité des résultats, l'une pour les articles et l'autre pour les comptes rendus. Ces grilles furent élaborées puis testées au moment de l'examen des numéros trimestriels de cinq années différentes, chacune de ces années étant choisie au hasard dans chaque groupe quinquennal: 1955-1959, 1960-1964, 1965-1969,

10. Voir pour exemple Fernand Harvey et Paul-André Linteau, "L'évolution de l'historiographie dans la «Revue d'histoire de l'Amérique française", 1947-1972. Aperçus quantitatifs", Revue d'histoire de l'Amérique française, vol. 26 , no 2 (septembre 1972), 163-183.

11. Bernard Berelson, Content Analysis in Communication Research, Glencoe, Free Press, 1952, p. 18. 
1970-1974 et 1975-1979.

Pour les articles, l'instrument ainsi constitué permet de recueillir les données suivantes:

- le sexe de l'auteur (masculin ou féminin);

- son statut (laiqque ou religieux) ;

- sa profession (bibliothécaire, technicien, professeur ou autres); dans ce cas lorsque l'auteur est à la fois professeur et bibliothécaire, seule la profession "professeur" est retenue ;

- sa formation (universitaire en bibliothéconomie, universitaire dans une autre discipline ou de collège); lorsqu'un individu possède une formation universitaire en bibliothéconomie et dans une autre discipline, nous ne retenons que la formation en bibliothéconomie :

- son lieu de travail (bibliothèques universitaire, nationale, de collège, scolaire, spécialisée et gouvernementale, publique, école de bibliothéconomie et département de techniques de la documentation ou autres);

- sa provenance (Montréal, Québec (ville), province, Ottawa ou extérieure au Québec à l'exception d'Ottawa).

L'autre partie de la grille concerne l'article et son contenu, soit le genre de l'article (opinion, état de la question ou recherche), la méthode utilisée (historique, descriptive, expérimentale, bibliographique ou ne s'applique pas), la longueur, le ou les sujets traités et le nombre de références infrapaginales. Nous devons préciser que nous considérons comme une référence infrapaginale tout renvoi à une monographie, à un périodique ou à tout autre document ou toute note personnelle de l'auteur. Sont donc exclues de ce calcul les bibliographies et les listes sélectives de titres accompagnant parfois les textes.

C'est évidemment au moment de déterminer le genre de l'article et la méthode choisie par les auteurs que nous avons dû procéder à des choix qualitatifs suite à la lecture de chacun des textes. Pour l'analyse des sujets traités par les articles, plus de trente thèmes ont été retenus. C'est délibérément qu'aucun regroupement de thèmes n'a été opéré puisque nous voulions obtenir ainsi le portrait le plus exact possible et vérifier l'hypothèse voulant qu'à un développement de la profession corresponde en concomitance une prolifération des sujets traités et une spécialisation de la littérature. De plus, nous n'avons pas hésité à utiliser plus d'un sujet pour décrire un article lorsque cela se révélait inéluctable. Notons également que chaque article correspond à une entrée distincte. Toutefois, dans le cas des articles rédigés par plusieurs auteurs, les caractéristiques de chacun des auteurs sont prises en compte.

La grille d'analyse des comptes rendus est construite à l'image de la précédente à l'exception de quelques détails que nous énumérerons. Ainsi, les mêmes variables nommées précédemment relativement aux auteurs d'articles sont requises et une liste identique de sujets est utilisée. Celle-ci nous sert cette fois à caractériser le contenu des volumes recensés. Les autres données qu'il nous importait de relever concernent les volumes analysés. Nous avons précisé le lieu d'édition Québec, France, États-Unis, Grande-Bretagne, Canada (autre que Québec) ou autres -, la langue d'édition - française, anglaise, bilingue ou autres - et le nombre de pages du compte rendu. Précisons que lorsque plus d'un volume est recensé dans un même compte rendu, les caractéristiques de chaque volume sont retenues.

Donc, nous avons effectué un dépouillement exhaustif de tous les numéros trimestriels du Bulletin de l'ACBLF/Documentation et bibliothèques sur une période de vingt-cinq ans (19551979), ce qui représente cent numéros analysés. Cette démarche nous a permis de retracer au total 826 articles qu'ont signés 797 auteurs; 84 articles sont anonymes. Puis, nous avons dénombré 435 comptes rendus rédigés par 425 auteurs; 18 comptes rendus ne sont pas signés.

Par rapport aux cinq périodes, ces chiffres se répartissent comme suit : nous comptons en 19551959117 articles signés par 108 auteurs dont 13 articles anonymes et 38 volumes ont fait l'objet d'un compte rendu par 33 auteurs. En 1960-1964, 270 articles parmi lesquels 45 sont sans auteur, ont été rédigés par 235 auteurs. De plus, 116 auteurs ont analysé 133 volumes. Au cours de la période 19651969, 172 articles ont été fournis par 164 auteurs sauf 13 articles qui n'étaient pas signés ; 89 auteurs ont recensé 87 volumes. Dans le quinquennat 19701974,161 articles, à l'exception de 10 anonymes, ont été écrits par 170 auteurs ; aussi, 93 volumes étaient analysés par 90 auteurs. Au cours de la période 1975-1979, 106 articles étaient livrés par 120 auteurs, hormis 3 articles non signés, et 97 auteurs recensaient 93 volumes.

L'examen de toutes ces contributions en fonction des variables déterminées a permis d'élaborer de nombreux tableaux que nous analyserons ultérieurement. Dans la plupart de ces graphiques et tableaux, les résultats sont exprimés en tenant compte des cinq périodes quinquennales déjà mentionnées. En plus de l'étude des auteurs et de leurs écrits, il nous est apparu essentiel de considérer un autre élément, soit la répartition des auteurs selon le nombre d'articles et de comptes rendus écrits par chacun d'eux. Cette observation donne lieu à deux autres tableaux qui permettront de vérifier si la plupart des contributions sont le lot d'un même groupe d'auteurs qui aurait largement collaboré à la revue. Peut-on parler d'un monopole exercé par un noyau restreint d'auteurs ou constaterons-nous une véritable ouverture de la revue à un nombre élevé de collaborateurs?

De ces divers tableaux et graphiques se dégagent des constatations qui mettent en lumière et solutionnent de nombreuses questions que nous nous étions posées bien avant d'entreprendre cette étude. Nous pouvons sans doute les résumer dans cette interrogation: les vingt-cinq années d'existence de notre revue témoignent-elles véritablement d'une évolution tant au niveau de sa conception, de son contenu, de son choix de 
collaborateurs qu'au niveau de la discipline et de la profession au Québec dont elle est nécessairement le reflet?

\section{Analyse des données}

\section{Les auteurs}

Pour cerner l'évolution de la revue, il nous importe de connaître les auteurs tout autant que les articles qu'ils ont écrits. Aussi, l'analyse de six variables se révèle primordiale, ces variables étant le sexe, le statut, la profession, la formation, le lieu de travail et la provenance géographique. Les graphiques qui suivent illustrent les résultats obtenus pour ces diverses caractéristiques.

Graphique 1 :

$\%$ Sexe des auteurs des articles

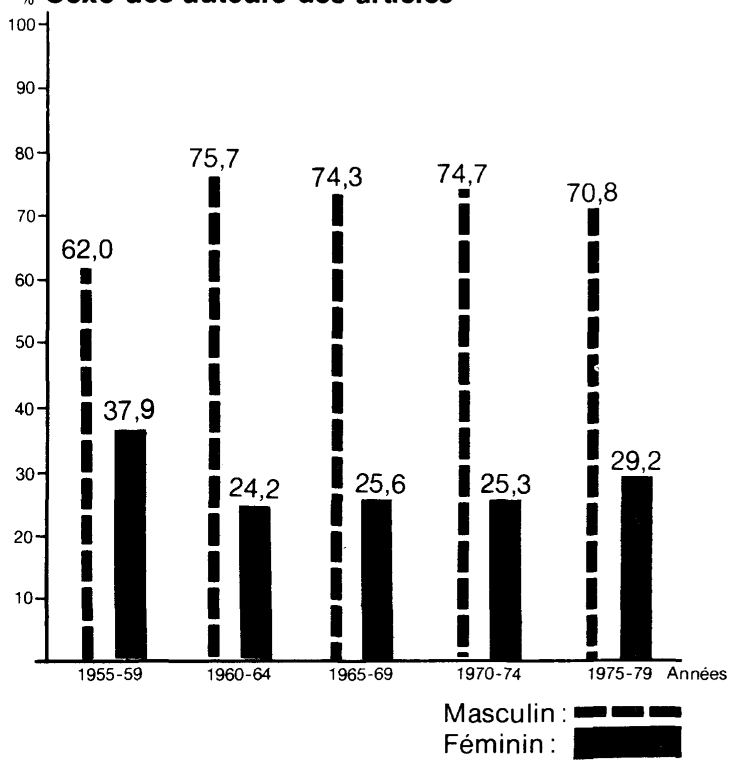

Graphique 2:

\% Sexe des auteurs des comptes rendus

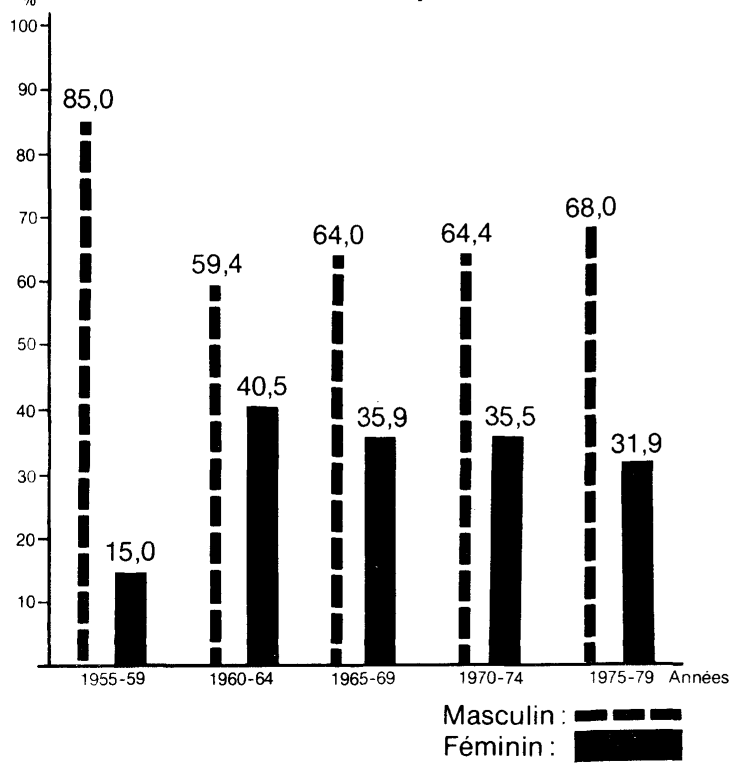

Nous savons que la profession de bibliothécaire recrute davantage de femmes que d'hommes. D'ailleurs, l'examen d'un échantillon de listes de membres de l'association ACBLF/ASTED nous le prouve sans conteste. Toutefois, l'écriture en bibliothéconomie est dans une grande proportion le fait des hommes, comme le démontrent les graphiques 1 et 2. En effet, une moyenne de $75 \%$ des collaborateurs sont des hommes quand il s'agit des articles. Même la décennie 1970 n'apporte pas de changement notable dans ces proportions. Nous présumions une remontée au niveau des contributions venant d'auteurs féminins, du moins pour la dernière période 1975-1979; celle-ci existe, mais elle est si minime qu'elle est à peine significative.

Graphique 3 :

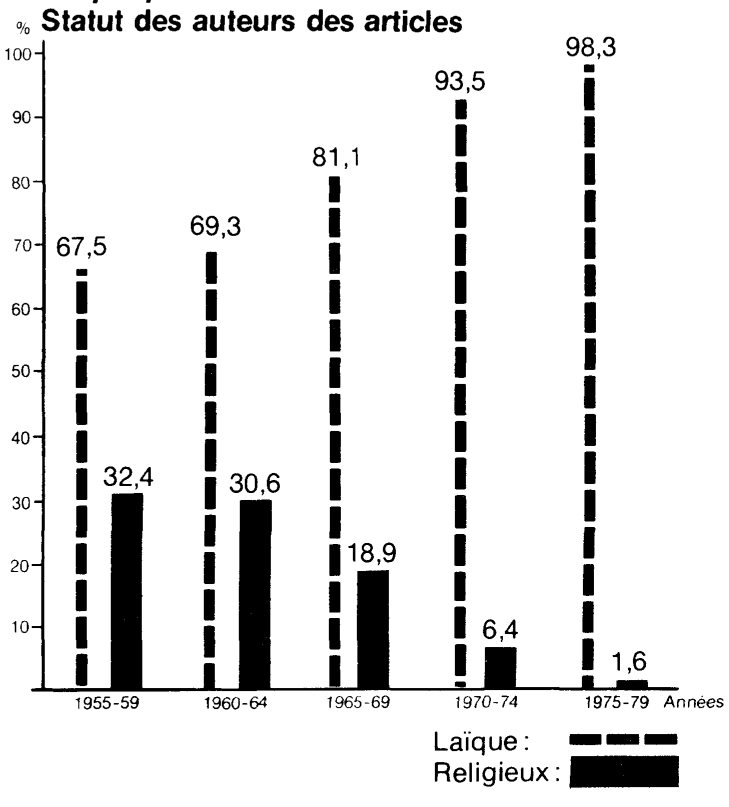

\section{Graphique 4 :}

$\%$ Statut des auteurs des comptes rendus

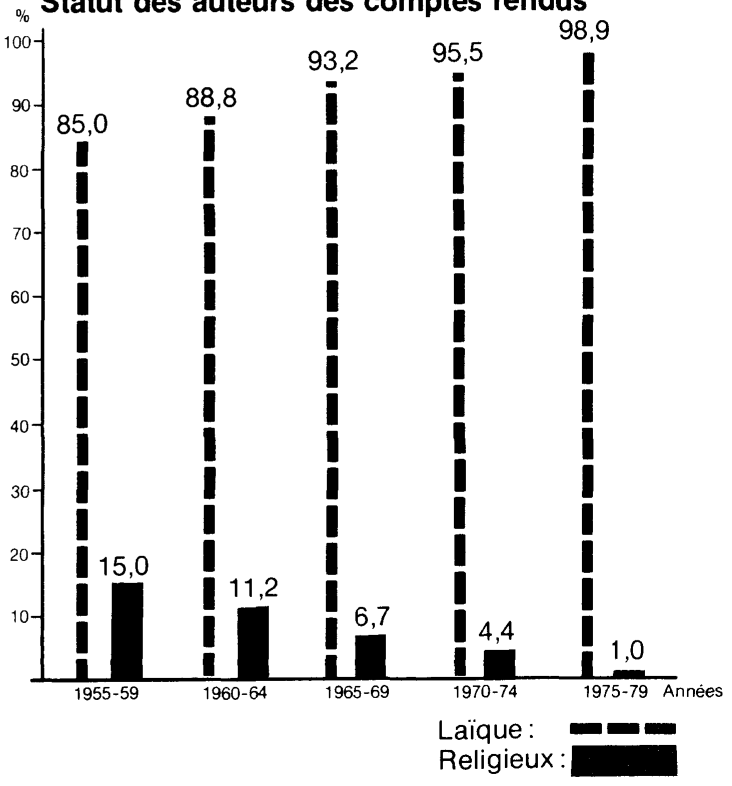


Il est par ailleurs étonnant de constater que les comptes rendus, longtemps considérés comme des collaborations secondaires par rapport aux articles qui composaient l'essentiel des sommaires, furent tout de même rédigés dans une proportion un peu plus élevée par des femmes.

Comment expliquer cet état de fait? Nous avons l'impression que les femmes, bien qu'elles adhèrent en plus grand nombre à l'Association, n'ont exercé et n'exercent que peu de leadership au sein de cette dernière ; elles sont malheureusement en minorité au sein des conseils d'administration. Ainsi, les activités de direction, d'orientation sont l'apanage des hommes tant au niveau de l'Association que dans les institutions où ils œuvrent. Les femmes manquent-elles de temps, d'intérêt ou de possibilités pour s'adonner à l'écriture?

La profession qui contribue à la revue est à l'image de la société québécoise : elle s'est laïcisée au milieu de la décennie 1960. N'eût été la collaboration assidue, en ce qui a trait aux articles, des pères Edmond Desrochers et Auguste-M. Morisset dans le reste de cette décennie, cette constatation aurait été beaucoup plus manifeste!

Or, c'est au cours de la décennie 1970 que la plupart des grands ténors religieux de la bibliothéconomie se retirent de la vie active de l'Association et de l'avant-scène de la profession. C'est à cette époque également que l'on recrute de nouveaux collaborateurs; cette relève est en majeure partie composée de jeunes bibliothécaires laïques nouvellement formés dans les écoles universitaires pendant la décennie 1960. D'ailleurs, le graphique 3 montre que les laïques représentent au-delà de $90 \%$ des auteurs d'articles dans la décennie 1970.

C'est donc uniquement pendant les années 1955-1964 que les religieux fournissent une collaboration importante à la revue en signant en moyenne $31,5 \%$ des articles. Rappelons que c'est à cette époque également que "les bibliothécaires les plus avertis et les plus actifs sont généralement des religieux qui élaborent la pensée de l'Association et en définissent les lignes d'action $\$ 12$.

Cependant, les religieux semblent moins attirés par l'activité de recension. Ils ne fourniront que $13,1 \%$ des comptes rendus entre 1955 et 1964 . Ceci s'explique: pendant ces années, les comptes rendus étaient en quelque sorte l'apanage des membres du comité de rédaction du Bulletin parmi lesquels on comptait très peu de religieux.

II ne faut pas se surprendre de constater que la revue, dont le principal intérêt est la bibliothéconomie, est écrite par des bibliothécaires, qu'ils soient praticiens ou théoriciens. Le monde de la bibliothéconomie québécoise a connu de nombreuses transformations depuis 1955. D'abord, en 1961, est fondée l'École de bibliothéconomie avec son corps professoral de carrière pour lequel il devenait intéressant et même important de collaborer à une revue professionnelle. Ainsi,

12. Gilbert Gagnon, « Les idéologies des bibliothécaires canadiens-français (1951-1968)", Documentation et bibliothèques, vol. 22, no 4 (décembre 1976), 171. depuis 1960, l'apport des professeurs de bibliothéconomie (de Montréal surtout et d'Ottawa) est assez constant au niveau des articles. Quant aux comptes rendus, les professeurs y ont contribué de façon significative dans la décennie 1960, mais beaucoup moins au cours des années 1970 . Ont-ils exercé pendant les années 1960 un rôle de suppléance dans ce domaine ou ont-ils plutôt volontairement mis l'accent sur les articles depuis 1970 pour répondre aux exigences du milieu universitaire? Ces deux énoncés expliquent dans une certaine mesure les données que nous livrent les graphiques 5 et 6 .
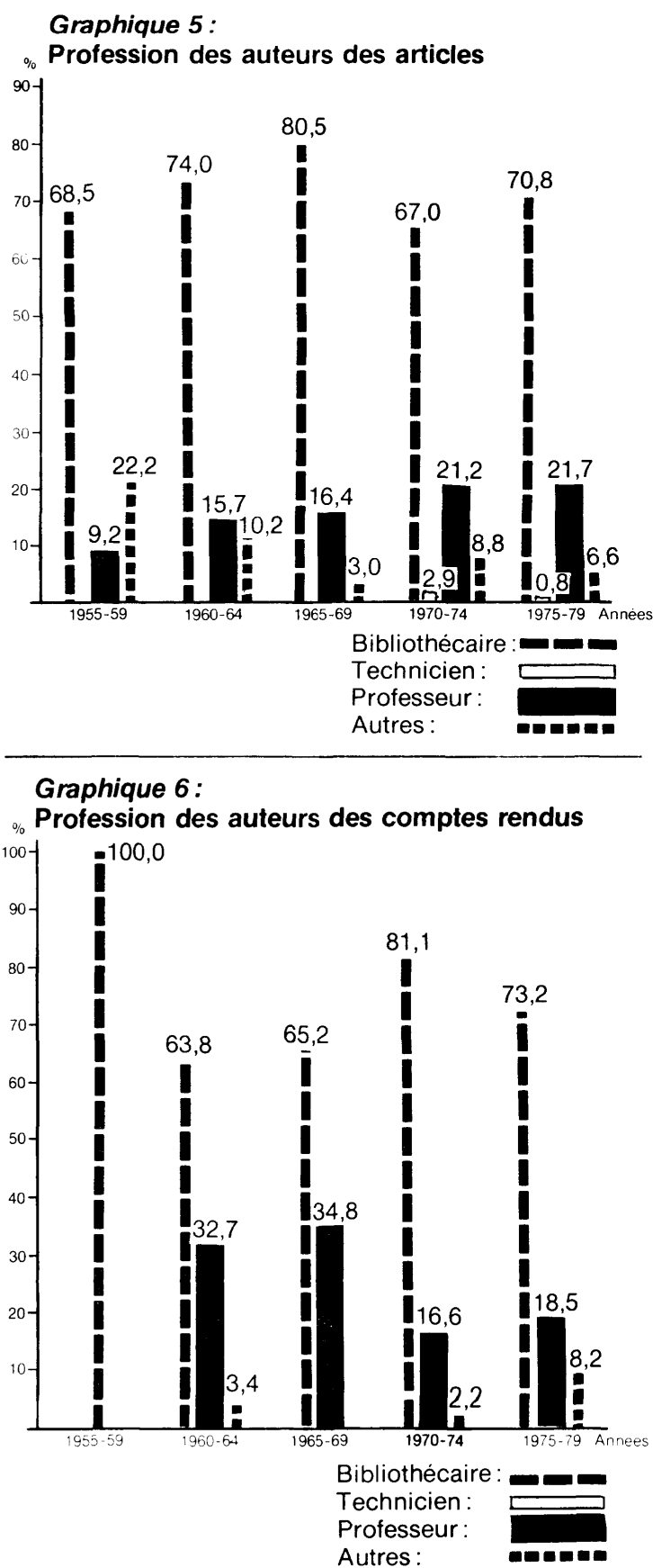
La première promotion de bibliotechniciens ou techniciens de la documentation n'arrive sur le marché du travail qu'en 1971. II n'est donc pas étonnant de ne relever aucune contribution de leur part avant 1970. Après 1970, leur apport est bien mince (en moyenne 1,85\% des articles et aucun compte rendu). Si l'on écarte le fait qu'ils sont peu nombreux, il ne reste pour expliquer ce phénomène que leur relative jeunesse, leur difficulté d'identification à l'Association et le peu d'importance qu'ils semblent accorder à la revue comme moyen de communication privilégié avec leurs pairs et les autres membres de la profession.

\section{Graphique 7 :}

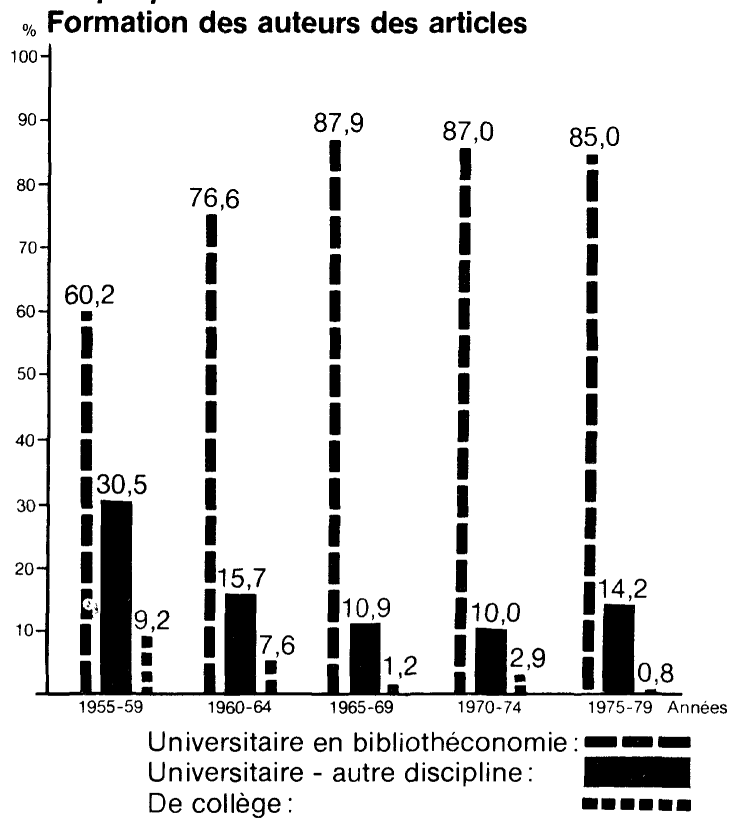

Graphique 8 :

$\%$ Formation des auteurs des comptes rendus

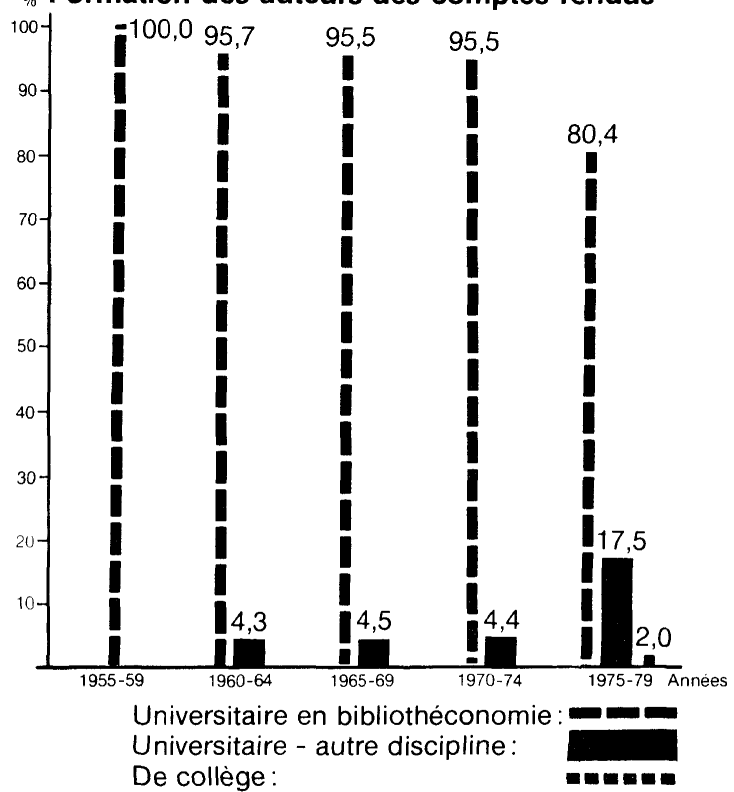

La catégorie «autres» réunit, en 1955-1959, des collaborateurs hétérogènes dont l'une des préoccupations est le monde du livre. De Pie XII à un éditeur en passant par des clercs intéressés aux questions pédagogiques ou au choix de livres, on pouvait lire à cette époque des auteurs de tout genre. A partir des années 1960, les objectifs de la revue se sont précisés et les auteurs virtuels au sein même de la profession se sont multipliés.

Les données si imposantes relatives à ceux qui possèdent une formation universitaire en bibliothéconomie à partir de 1960 s'expliquent du fait que cette catégorie rassemble des bibliothécaires et des professeurs dont la majeure partie enseigne à l'École de bibliothéconomie (graphiques 5 et 6 ).

Avant la fondation de l'École de bibliothéconomie (1955-1959), de nombreux bibliothécaires qui collaboraient à la revue possédaient seulement une formation universitaire dans une autre discipline ou une formation de collège. En effet, «la majorité des membres de l'Association n'ont pas un diplôme universitaire reconnu en bibliothéconomie »13. Les années 1960 amènent une clarification dans le titre et la formation reçue. Les chiffres désignant ceux qui possèdent une formation universitaire dans une autre discipline, en hausse pour 1975-1979, sont d'une nature différente; ce sont des collaborations de type interdisciplinaire qui amorcent sans doute une tendance.

D'autre part, il nous faut distinguer les auteurs qui ont reçu une formation de collège avant 1970 de ceux de la décennie 1970. Avant 1970, une portion du personnel des bibliothèques possédait des études classiques (souvent le baccalauréat ès arts) tandis qu'après 1970, on se réfère davantage à ces nouveaux diplômés de collège venus de l'option "techniques de la documentation».

Au niveau des comptes rendus, nous remarquons pour le dernier quinquennat une collaboration accrue de professeurs spécialistes en d'autres disciplines à qui l'on demande surtout des recensions d'ouvrages de référence spécialisés ou de bibliographies dans leur domaine. Encore une fois les individus ne possédant qu'une formation de collège ne sont à peu près jamais sollicités pour la rédaction de comptes rendus: leur apport ne représente que $2 \%$ de l'ensemble des contributions du quinquennat 1975-1979 et ils sont totalement absents dans les années précédentes.

En 1955-1959, trois groupes dominent; les auteurs travaillant dans les bibliothèques universitaires, spécialisées et publiques contribuent dans une proportion de $53,6 \%$ aux articles. II faut remarquer que plus du quart $(26,8 \%)$ des auteurs ne travaillent pas dans une bibliothèque. Ceux-ci s'identifient au groupe «autres" que nous avons mentionné au moment de l'analyse de la formation. Ce sont des « amis du livre», des clercs, des éditeurs ou autres. Le quinquennat 1960-1964 connaît l'émergence des bibliothèques de collège et l'arrivée des professeurs de l'École de bibliothéco- 
Tableau 1: Lieu de travail des auteurs des articles

\begin{tabular}{|l|r|r|r|r|c|}
\hline & $1955-59$ & $1960-64$ & $1965-69$ & $1970-74$ & $1975-79$ \\
& $\%$ & $\%$ & $\%$ & $\%$ & $\%$ \\
\hline 1. - Bibliothèque universitaire & 13,9 & 19,1 & 32,3 & 29,4 & 26,7 \\
2. - Bibliothèque nationale & 0,9 & 2,5 & 5,5 & 8,2 & 13,3 \\
3. - Bibliothèque de collège & 9,3 & 13,6 & 12,8 & 4,7 & 6,7 \\
4. - Bibliothèque scolaire & 6,5 & 6,0 & 4,3 & 2,4 & 3,3 \\
5. - Bibliothèque spécialisée & 21,2 & 23,0 & 7,3 & 13,5 & 6,7 \\
6. - Bibliotheqque publique & 18,5 & 9,8 & 17,1 & 8,8 & 11,6 \\
7. - Ecole de bibliothéconomie ou département & 2,7 & 15,7 & 14,6 & 23,0 & 17,5 \\
de techniques de la documentation & & & & & \\
8. - Autres & 26,8 & 10,2 & 6,1 & 10,0 & 14,2 \\
\hline
\end{tabular}

Tableau 2: Lieu de travail des auteurs des comptes rendus

\begin{tabular}{|l|r|r|r|r|r|}
\hline & \multicolumn{1}{|c|}{$\begin{array}{c}1955-59 \\
\%\end{array}$} & $\begin{array}{c}1960-64 \\
\%\end{array}$ & $\begin{array}{c}1965-69 \\
\%\end{array}$ & $\begin{array}{c}1970-74 \\
\%\end{array}$ & $\begin{array}{c}1975-79 \\
\%\end{array}$ \\
\hline 1. - Bibliothèque universitaire & 6,0 & 28,4 & 36,0 & 23,3 & 31,9 \\
2. - Bibliothèque nationale & - & 0,9 & 4,5 & 8,2 & 7,2 \\
3. - Bibliothèque de collège & 63,6 & 10,3 & 5,6 & 8,9 & 5,2 \\
4. - Bibliothèque scolaire & 3,0 & 2,6 & 5,6 & 4,4 & 3,1 \\
5. - Bibliothèque spécialisée & 24,2 & 9,5 & 3,4 & 16,7 & 19,6 \\
6. - Bibliothèque publique & 3,0 & 12,1 & 10,1 & 15,5 & 7,2 \\
7 - Ecole de bibliothéconomie ou département & - & 32,7 & 33,7 & 15,5 & 11,3 \\
$\quad$ de techniques de la documentation & & & & & \\
8. - Autres & - & 3,4 & 1,1 & 6,7 & 14,7 \\
\hline
\end{tabular}

nomie. C'est dans les bibliothèques universitaires, de collège, spécialisées et à l'École de bibliothéconomie que l'on retrouve $70,7 \%$ des auteurs. En 1965-1969, les bibliothèques universitaires prennent nettement la tête du peloton avec le tiers des auteurs (32,3\%). Dans le quinquennat 1975-1979, la ventilation des pourcentages est beaucoup plus variée. Les deux bibliothèques nationales fournissent davantage d'auteurs $(13,3 \%)$ et les bibliothèques universitaires plus du quart $(26,7 \%)$. L'École de bibliothéconomie et les départements de techniques de la documentation avec $17,5 \%$ des auteurs et les bibliothèques publiques $(11,6 \%)$ forment à nouveau un groupe dynamique.

Sur un plan synchronique, nous remarquons que les bibliothèques scolaires ont fourni un faible apport $(4,5 \%$ des articles en moyenne) ne dépassant jamais $6,5 \%$ des contributions; voilà une constatation qui reflète l'état des bibliothèques du monde scolaire au Québec.

Au niveau des comptes rendus, l'apport très élevé venant des bibliothèques de collège, en 19551959, s'e xplique par la contribution importante d'un seul et reeme auteur. Pour 1960-1969, à peine $1 \%$ des $m \epsilon$ bres de la profession que sont les professe is de l'École de bibliothéconomie fournit le tiers des comptes rendus. On remarque à nouveau que les auteurs venant des bibliothèques universitaires occupent de loin le premier rang. Au cours de la période 1975-1979, nous constatons que les auteurs dits " autres " fournissent $14,2 \%$ des articles et $14,7 \%$ des comptes rendus. Ces chiffres en hausse par rapport aux périodes précédentes s'expliquent aussi par la collaboration accrue de professeurs d'autres départements et démontrent bien le début d'une interdisciplinarité et la fin du cloisonnement d'une revue rédigée par les bibliothécaires seuls.

L'intérêt d'utiliser la variable "provenance géographique» réside dans le fait que nous voulions évaluer l'importance de l'apport des auteurs de l'île de Montréal.

Une constante se dégage nettement pour toute la période; les auteurs d'articles de bibliothéconomie dans notre revue sont montréalais dans une proportion de $51,9 \%$ (en moyenne). On dénote une stabilité à tous les autres niveaux. Québec, notamment par sa bibliothèque universitaire, ses bibliothèques administratives, la Bibliothèque de l'Assemblée nationale, fournit une bonne part des auteurs. La même remarque vaut pour Ottawa avec la présence des bibliothécaires francophones dans les diverses bibliothèques gouvernementales, universitaires et à la Bibliothèque nationale.

Les auteurs de l'extérieur de Montréal et de Québec ont également fourni une contribution dans une proportion voisine de celle de Québec. Les collaborations "extérieures au Québec", de 1955 à 1969 , viennent pour la plupart d'auteurs francophones résidant au Canada. Cependant, depuis 1970, ce groupe se compose principalement de spécialistes européens. 
Tableau 3: Provenance géographique des auteurs des articles

\begin{tabular}{|c|c|c|c|c|c|}
\hline & $\begin{array}{c}1955-59 \\
\%\end{array}$ & $\begin{array}{c}1960-64 \\
\%\end{array}$ & $\begin{array}{c}1965-69 \\
\%\end{array}$ & $\begin{array}{c}1970-74 \\
\%\end{array}$ & $\begin{array}{c}1975-79 \\
\%\end{array}$ \\
\hline 1. - Montréal & 57,4 & 52,0 & 43,3 & 53,5 & 53,3 \\
\hline 2. - Québec (ville) & 6,5 & 14,0 & 15,8 & 18,2 & 16,7 \\
\hline 3. - Province & 19,4 & 17,4 & 17,1 & 12,4 & 14,1 \\
\hline 4. - Ottawa & 9,2 & 12,7 & 13,4 & 12,4 & 10,0 \\
\hline 5. - Extèrieure au Québec (à l'exception d'Ottawa) & 7,4 & 3,8 & 10,3 & 3,5 & 5,8 \\
\hline
\end{tabular}

Tableau 4 : Provenance géographique des auteurs des comptes rendus

\begin{tabular}{|c|c|c|c|c|c|}
\hline & $\begin{array}{c}1955-59 \\
\%\end{array}$ & $\begin{array}{c}1960-64 \\
\%\end{array}$ & $\begin{array}{c}1965-69 \\
\%\end{array}$ & $\begin{array}{c}1970-74 \\
\%\end{array}$ & $\begin{array}{c}1975-79 \\
\%\end{array}$ \\
\hline 1. - Montréal & 93,9 & 87,9 & 65,2 & 68,9 & 62,9 \\
\hline 2. - Québec (ville) & 3,0 & 3,4 & 7,9 & 14,4 & 20,6 \\
\hline 3. - Province & - & 3,4 & 21,3 & 12,2 & 8,2 \\
\hline 4. - Ottawa & - & 5,2 & 5,6 & 4,4 & 8,2 \\
\hline 5. - Extérieure au Québec (à l'exception d'Ottawa) & 3,0 & - & - & - & - \\
\hline
\end{tabular}

L'activité de recension s'accomplit essentiellement à Montréal et à Québec ( $85,6 \%$ en moyenne) pour l'ensemble des périodes. Comme le montre le tableau 9: Lieu d'édition des volumes recensés, une très grande majorité des volumes analysés sont québécois et de langue française, ils sont donc recensés par des auteurs d'ici. Assistons-nous à un excès d'endogamie intellectuelle?

\section{Les articles}

Les 3183 pages d'articles publiés au cours du quart de siècle de parution forment une partie essentielle du message véhiculé par la revue. II nous est apparu important de faire un relevé de tous les thèmes traités au cours de ces années, de façon à faire ressortir la variation des champs d'intérêts professionnels. La discipline et la profession ont évolué considérablement, au Québec particulièrement. Le contenu de la revúe en est-il un reflet fidèle?

Sur cet ensemble de données, il nous faut poser à la fois un regard synchronique et un regard diachronique pour relever les éléments les plus éloquents. En 1955-1959, quatre thèmes retiennent davantage l'attention: édition et librairie, services techniques, livres et lecture pour les enfants et surtout les associations de bibliothèques; la jeune Association fonde un Bulletin pour informer ses membres et resserrer les structures de l'organisme. C'est un bulletin d'information, un forum pour les présidents de l'Association et des comités. La normalisation des services techniques, préoccupation manifeste, l'emporte sur les services publics et les collections, questions pourtant essentielles pour un service documentaire. En 1960-1965, la formation $\mathrm{du}$ bibliothécaire et les problèmes d'associations occupent $52,2 \%$ de l'espace de la revue. La fondation de l'École de bibliothéconomie explique cet intérêt. Par ailleurs, les problèmes d'information et de fonctionnement de l'Association couvrent près de la moitié des livraisons; cet état ne pouvait perdurer. Cela amènera en 1965 la fondation des Nouvelles de l'ACBLF, et nous en constatons les résultats au cours du quinquennat 1965-1969, avec la baisse de $47,1 \%$ à $19,4 \%$ des articles sur ce thème. Les modifications du programme de baccalauréat et la préparation du programme de maîtrise à l'École, l'instauration des programmes de bibliotechnique dans les collèges, le passage à Montréal de la Fédération internationale des associations des bibliothèques en 1967 et la rénovation des bibliothèques universitaires sont les questions dominantes durant les années 19651969. Ce qui ressort, en 1970-1974, ce sont les problèmes d'administration des bibliothèques, d'automatisation et de services publics ; la formation des bibliothécaires, à cause du nouveau programme de maîtrise, retient encore l'attention des auteurs. De 1975 à 1979, nous notons une hausse d'intérêt pour l'administration, la montée, déjà notée en 1970-1974, des services techniques, la place importante, due à une chronique régulière, des livres et lecture pour enfants et une part réelle accordée aux problèmes des sciences de l'information et d'informatique documentaire (12,2\% pour les catégories 15 à 18). Les auteurs prennent aussi conscience du rôle de l'État dans une politique de bibliothèques, et en particulier en ce qui concerne les bibliothèques publiques.

D'autre part, au cours de ces vingt-cinq années, l'étude des fondements, essentielle à une discipline en formation, a été peu retenue. Contrairement à nos présomptions, la censure ou l'orientation des lectures n'a pas fait l'objet de longs 
Tableau 5 : Distribution des sujets des articles

\begin{tabular}{|c|c|c|c|c|c|c|}
\hline & $\begin{array}{c}1955-59 \\
\%\end{array}$ & $\begin{array}{c}1960-64 \\
\%\end{array}$ & $\begin{array}{c}1965-69 \\
\%\end{array}$ & $\begin{array}{c}1970-74 \\
\%\end{array}$ & $\begin{array}{c}1975-79 \\
\%\end{array}$ & $\begin{array}{c}\text { Total } \\
1955-79 \\
\%\end{array}$ \\
\hline \multicolumn{7}{|l|}{ Sujets } \\
\hline $\begin{array}{l}\text { 1. Fondements, théorie et philosophie de la } \\
\text { bibliothéconomie }\end{array}$ & 1,5 & 1,5 & 2,8 & 2,8 & 2,7 & 2,25 \\
\hline $\begin{array}{l}\text { 2. Formation (initiale et continue) du } \\
\text { bibliothécaire }\end{array}$ & 1,5 & 5,1 & 10,9 & 8,5 & 1,4 & 5,48 \\
\hline 3. Formation du technicien & - & 0,9 & 3,3 & 4,0 & 1,4 & 1,92 \\
\hline 4. Formation (autres) & - & - & 0,5 & 0,4 & - & 0,18 \\
\hline 5. Bibliothéconomie comparée et internationale & 3,1 & 2,7 & 4,7 & 3,2 & 2,7 & 3,28 \\
\hline 6. Censure & 2,3 & 0,3 & - & - & - & 0,52 \\
\hline 7 Histoire du livre et des bibliothèques & 2,3 & 2,4 & 5,3 & 0,8 & 2,0 & 2,56 \\
\hline 8. Édition et librairie & 5,4 & 1,2 & 2,4 & 2,1 & 1,4 & 2,3 \\
\hline 9. Administration des bibliothèques & 0,8 & 4,2 & 4,7 & 7,3 & 9,5 & 5,3 \\
\hline 10. Personnel & 0,8 & 0,9 & 1,9 & 1,6 & 2,0 & 1,44 \\
\hline 11. Architecture et aménagement & - & 0,6 & 1,4 & 1,2 & - & 0,64 \\
\hline $\begin{array}{l}\text { 12. Services techniques (classification, } \\
\text { catalogage, indexation, acquisitions) }\end{array}$ & 5,4 & 3,0 & 3,8 & 5,3 & 8,2 & 5,14 \\
\hline $\begin{array}{l}\text { 13. Services publics (prêt, référence, formation } \\
\text { des usagers) }\end{array}$ & - & 0,6 & 0,5 & 4,5 & 4,1 & 1,94 \\
\hline 14. Collections (choix, coordination, élagage) & - & 0,6 & 0,5 & 0,4 & 3,4 & 0,98 \\
\hline 15. Sciences de l'information (sujets relatifs aux) & & - & 0,9 & 0,8 & 2,7 & 0,88 \\
\hline $\begin{array}{l}\text { 16. Informatique et automatisation des } \\
\text { bibliothèques }\end{array}$ & & 0,3 & 3,3 & 6,5 & 5,4 & 3,1 \\
\hline 17. Banques et bases de données & & - & - & 1,2 & 1,4 & 0,72 \\
\hline 18. Réseaux & - & 0,3 & - & 0,4 & 2,7 & 0,68 \\
\hline 19. Documentation audiovisuelle & 3,1 & 0,6 & 1,4 & 1,2 & 2,0 & 1,66 \\
\hline 20. Publications officielles & - & 0,3 & - & 1,2 & 1,4 & 0,58 \\
\hline 21. Périodiques & 1,5 & - & 1,4 & 0,4 & 1,4 & 0,94 \\
\hline 22. Bibliographie (générale et spécialisée) & 1,5 & 0,9 & 2,8 & 1,2 & - & 1,28 \\
\hline 23. Ouvrages de référence & - & - & - & - & - & \\
\hline $\begin{array}{l}\text { 24. Livres et lecture des enfants et des } \\
\text { adolescents }\end{array}$ & 9,3 & 2,1 & - & 4,9 & 8,9 & 5,04 \\
\hline 25. Milieu et usagers & - & 0,3 & - & 2,1 & 2,7 & 1,02 \\
\hline 26. Statistiques & - & 1,8 & - & 1,6 & 1,4 & 0,96 \\
\hline $\begin{array}{l}27 \text { Associations de bibliothèques et de } \\
\text { bibliothécaires }\end{array}$ & 31,0 & 47,1 & 19,4 & 7,8 & 3,4 & 21,74 \\
\hline 28. L'État et les bibliothèques & - & 1,2 & - & 2,4 & 5,4 & 1,8 \\
\hline 29. Archives & - & 0,3 & 0,5 & - & 0,7 & 0,3 \\
\hline 30. Autres & 7,0 & 2,7 & 1.4 & 3,7 & 1,4 & 3,24 \\
\hline \multicolumn{7}{|l|}{ Genres de bibliothèques } \\
\hline 1. Nationale & 1,5 & - & 3,3 & 2,1 & 1,4 & 1,66 \\
\hline 2. Universitaire & 3,1 & 0,9 & 5,3 & 4,5 & 4,8 & 3,72 \\
\hline 3. De collège & 0,8 & 4,5 & 4,7 & 1,6 & 1,4 & 2,6 \\
\hline $\begin{array}{l}\text { 4. Spécialisée et centre de documentation } \\
\text { (y compris bibliothèque gouvernementale) }\end{array}$ & 7,0 & 2,7 & 2,8 & 3,2 & 2,0 & 3,54 \\
\hline 5. Scolaire & 3,1 & 3,3 & 4,7 & 4,5 & 2,7 & 3,66 \\
\hline 6. Publique & 7,8 & 6,6 & 5,3 & 6,5 & 8,1 & 6,86 \\
\hline
\end{tabular}


débats au cours de la première décennie de la revue. Services publics et milieu et usagers (catégories 13 et 25), thèmes apparentés, ne retiennent que 2,96\% de l'espace pendant ces vingt-cinq ans: ils constituent pourtant l'un des piliers du service documentaire. Nous pourrions faire des remarques semblables pour les collections et les divers genres de documents $(6,12 \%$ pour les catégories 14 et 19 à 23).

Il est intéressant de remarquer que, pendant la période étudiée, $22 \%$ des articles portaient sur des genres de bibliothèques. Tous les genres ont été abordés, selon l'intérêt du moment; par exemple, bibliothèque nationale en 1965-1969, à cause de la création de la Bibliothèque nationale du Québec, bibliothèque universitaire, à cause des transformations en 1960-1965, bibliothèque de collège, lieu de grand dynamisme dans la décennie 1960, bibliothèque scolaire, qui attendait beaucoup du Rapport Parent au cours de la même décennie, bibliothèque publique, pour laquelle l'intérêt a été assez constant, même si l'on remarque une montée d'intérêt à cause du mémoire de programme du ministère des Affaires culturelles.

Enfin, plus nous approchons de la période présente, plus nous remarquons une diversification des sujets abordés concomitante à une spécialisation du traitement du contenu.

Le tableau 6 et les graphiques 9 et 10 , contrairement à tous les autres, sont d'ordre qualitatif. L'exposé de points de vue, l'énoncé de faits ou situations, l'état de la question sur un problème, la recherche originale, en un mot la structuration et l'avancement d'une discipline requièrent l'utilisation d'une méthode. beaucoup emprunté, depuis l'École de Chicago dans les années 1930, aux méthodes des sciences sociales et que la méthode descriptive s'était imposée fortement dans l'élaboration d'un corpus disciplinaire autonome. C'est le cas pour notre revue depuis les débuts ; cette ascension s'accentue après 1970. Parallèlement, le cadre expérimental, créateur s'il en est de connaissances et de théories nouvelles, ne ressort véritablement qu'après 1975. L'avenir nous dira si la méthode expérimentale poursuivra cette poussée. Par ailleurs, la chute des articles sans méthode se fait au profit des articles de nature descriptive. S'il demeure encore $10,4 \%$ de ces articles, l'on sent tout de même que les points de vue sont davantage documentés et font appel de plus en plus à des portions de méthodologie descriptive.

En corollaire aux méthodes utilisées, nous pouvons distinguer trois genres d'articles; les articles d'opinion reflètent la position ou les convictions d'un individu ou d'un groupe, les articles d'état de la question font le point sur un problème donné, d'une manière approfondie et documentée, tandis que les articles de recherche apportent vraiment des connaissances nouvelles.

De l'opinion qui a dominé les années 1955 à 1969, nous en sommes depuis 1970 à l'état de la question, étape sans doute de transition avant d'en arriver aux articles de recherche. La principale remarque que nous pouvons porter sur le graphique 9 est que nous sommes au seuil de la recherche, mais que nous n'y sommes pas encore entrés vraiment.

Nous avons noté, dans notre introduction, la transformation notable que représentait la mutation

Tableau 6: Méthode utilisée par les auteurs des articles

\begin{tabular}{|c|c|c|c|c|c|}
\hline & $\begin{array}{c}1955-59 \\
\%\end{array}$ & $\begin{array}{c}1960-64 \\
\%\end{array}$ & $\begin{array}{c}1965-69 \\
\%\end{array}$ & $\begin{array}{c}1970-74 \\
\%\end{array}$ & $\begin{array}{c}1975-79 \\
\%\end{array}$ \\
\hline 1 - Historique & 5,1 & 9,2 & 4,6 & 2,5 & 3,8 \\
\hline 2. - Descriptive & 47,0 & 60,7 & 61,6 & 77.6 & 76,4 \\
\hline 3. - Expérimentale & - & - & 0,6 & 0,6 & 8,5 \\
\hline 4. - Bibliographique & 0,8 & 1,8 & 4,6 & 3,7 & 0,9 \\
\hline 5. - Ne s'applique pas & 47,0 & 28,2 & 28,5 & 15,5 & 10,4 \\
\hline
\end{tabular}

En bibliothéconomie, les méthodes employées sont multiples. Nous avons spécifié pour chacun des articles la méthode, historique, descriptive, expérimentale ou bibliographique, quand celui-ci en fait usage à quelque degré que ce soit. Quand nous ne décelions aucune méthode, nous assignions la catégorie "ne s'applique pas ».

Jusqu'en 1970, le Bulletin de l'ACBLF faisait grande place à l'opinion et à la nouvelle, lesquelles ne nécessitaient l'utilisation d'aucune méthode. Par ailleurs, par les traités et les articles de périodiques sur les méthodes de recherche dans notre discipline, nous savions que le développement des méthodes de recherche en bibliothéconomie avait du Bulletin de I'ACBLF en Documentation et bibliothèques et les facteurs que le comité de rédaction avait invoqués alors en faveur de ce changement.

Nous voulions vérifier si, dans la réalité, ces deux périodes 1955-1972 et 1973-1979 présentaient un portrait différent. De $57,2 \%$ pendant la première période, les articles d'opinion baissent à $25,4 \%$ pendant la deuxième, tandis que les articles d'état de la question et de recherche passent de $42,8 \%$ à $74,5 \%$; nour remarquons que le pourcentage des articles dits de recherche a quadruplé de 1955-1972 à 1973-1979. L'évolution perçue dans l'examen de la revue est, sans doute, celle du développement de la 
discipline au Québec au cours du dernier quart de siècle.

Deux éléments peuvent de plus corroborer l'évolution déjà mise en évidence : le nombre moyen de pages par article et le nombre moyen de références infrapaginales par article.

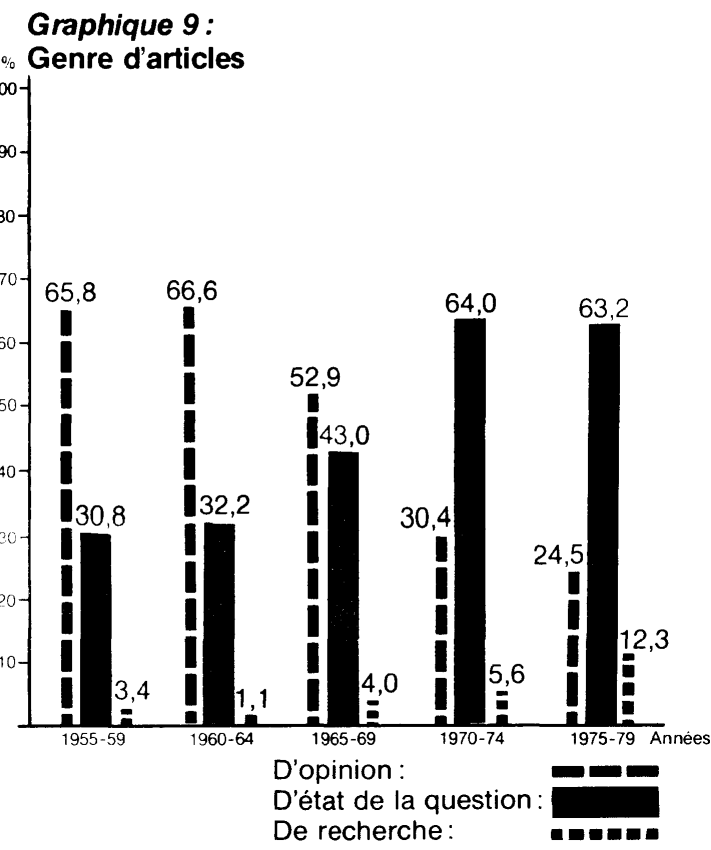

\section{Graphique 10:}

Comparaison des genres d'articles

Bulletin de l'ACBLF (1955-72)

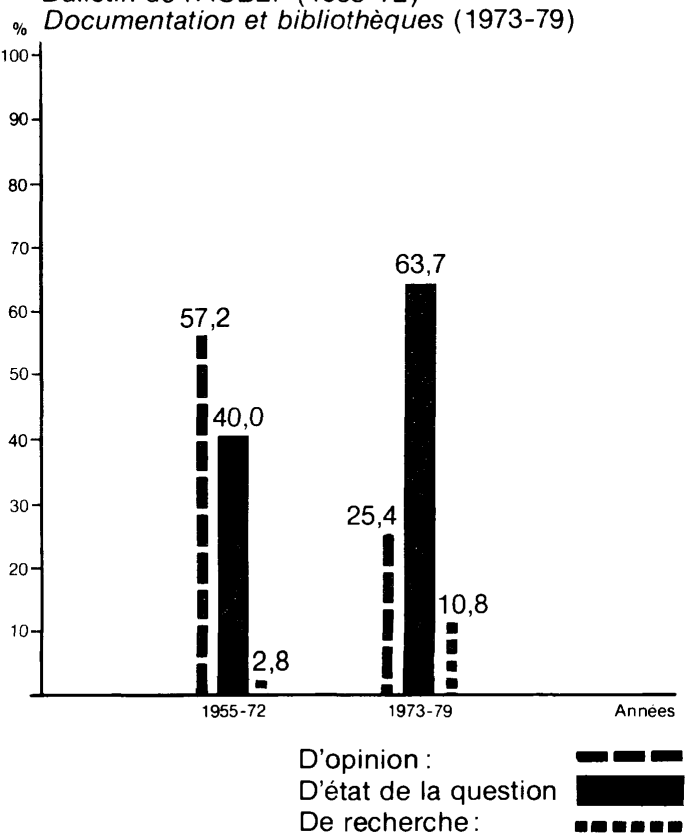

Un article détient un contenu, une méthode et nécessite aussi un espace pour présenter son message d'une manière explicite et articulée. II va de soi qu'un article d'opinion requiert moins d'espace pour l'expression des idées qu'un article de recherche ou d'état de la question pour la présentation des données et l'exposé des conclu-

Graphique 11:

Nombre moyen de pages par article Pages

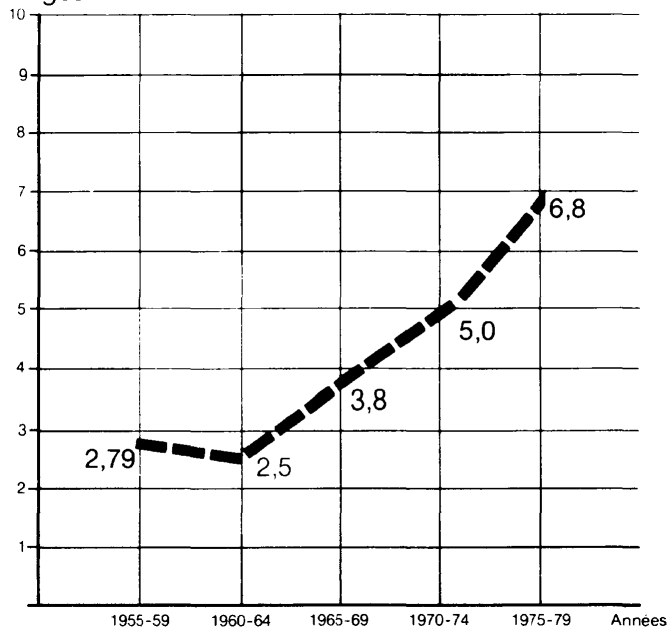

sions. Cette évolution des genres d'articles devait donc se concrétiser au plan spatial. Effectivement, l'espace requis par un article n'a cessé d'augmenter substantiellement dans les années 1960 et particulièrement depuis l'énoncé d'une nouvelle politique de rédaction en 1963. On peut s'attendre à ce que le niveau atteint, au cours du dernier quinquennat, soit un plateau qui se maintiendra dans les années à venir.

L'étude des citations est en voie de devenir un domaine d'étude privilégié en sciences de l'information ${ }^{14}$. Nos ambitions sont beaucoup plus modestes. Pour nous, il ne s'agit pas de faire une étude scientifique des fréquences des citations, ni de leur distribution géographique ou linguistique. II importait de quantifier, par période, le nombre de références infrapaginales par article et d'en visualiser le développement.

Le graphique 12 montre très nettement une coupure au tournant des années 1970. De 19551959 à 1965-1969, nous passons d'un nombre moyen de références de 0,64 à un nombre moyen de 1,78 références infrapaginales. En revanche, de 1965-1969 à 1975-1979, nous voyons un bond de 1,78 à 10,8. Ce changement est très significatif. Avec

14. Voir notamment: Yakov M. Rabkin, «Quelques aspects scientométriques de la documentation scientifique ", Argus, vol. 9, nos 5-6 (septembre-décembre 1980), 165-168; l'article déjà cité de Donald J. Lehnus, "JEL, 1960-1970 ", Journal of Education for Librarianship. Nous pensons de plus aux nombreux travaux d'Eugene Garfield sur le sujet. 
Graphique 12 :

Nombre moyen de références

infrapaginales par article

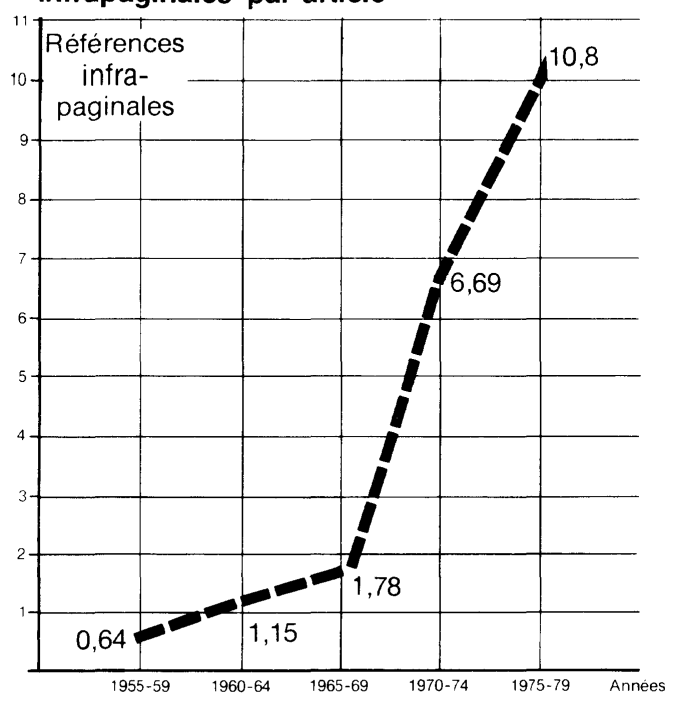

l'emploi de méthodes de recherche et en plus de l'augmentation d'espace pour chaque article, cette hausse des appuis bibliographiques, cet élargissement de la base documentaire représentent une modification importante et viennent confirmer les remarques faites lors de l'examen des genres d'articles. De plus, nous croyons que cette ascension devrait se poursuivre surtout si la bibliothéconomie québécoise doit donner lieu à davantage de recherche originale.

On peut se demander si la collaboration au Bulletin de l'ACBLF/Documentation et bibliothèques a été le seul fait d'un petit groupe d'auteurs qui s'adonnent au plaisir de l'écriture.

Tableau 7 : Répartition articles/auteurs (1955-1979)

\begin{tabular}{|cc|}
\hline Nombre d'articles & Nombre d'auteurs \\
\hline 1 & 221 \\
2 & 67 \\
3 & 20 \\
4 & 8 \\
5 & 5 \\
6 & 6 \\
7 & 8 \\
8 & 5 \\
9 & 4 \\
10 à 14 & 6 \\
15 à 19 & 2 \\
20 et plus & 2 \\
\hline
\end{tabular}

Une répartition du nombre d'articles en fonction du nombre d'auteurs prouve nettement qu'il n'en est rien. Trois cent cinquante-trois auteurs différents ont signé 742 articles au cours de la période étudiée. De ce nombre, 221 collaborateurs n'ont écrit qu'un seul article, 67 ont signé deux articles et 20 ont rédigé trois articles. Ces seuls chiffres illustrent hors de tout doute la large collaboration à la revue.

Quatre auteurs ont signé plus de 15 articles chacun (Réal Bosa 17, Auguste-M. Morisset 19, Laurent-G. Denis 20 et Edmond Desrochers 26). Faut-il souligner que ces auteurs les plus prolifiques ont déjà détenu la présidence de l'Association ? Nous nous demandons alors s'ils ont beaucoup écrit parce qu'ils étaient présidents ou bien étaient-ils présidents parce qu'ils avaient atteint une certaine notoriété par la rédaction d'articles ${ }^{15}$. Selon nous, on peut relier jusqu'à un certain point la prolificité des auteurs à l'importance des responsabilités que plusieurs d'entre eux ont assumé au sein de l'Association.

II est à remarquer que la dissémination des auteurs s'accentue au cours de la décennie 1970; ceci est à notre avis un signe de santé pour l'avenir d'une revue.

\section{Les comptes rendus}

Secteur souvent négligé, les comptes rendus constituent une activité scientifique essentielle dans l'avancement d'une discipline et une partie importante dans l'évaluation d'une revue ${ }^{16}$. Nous nous sommes servis pour la ventilation des sujets des volumes recensés de la même grille thématique qui avait été élaborée pour les sujets d'articles.

Les principales conclusions d'ensemble qui se dégagent de l'examen du tableau 8 sont essentiellement les suivantes: les ouvrages consacrés aux services techniques ont fait l'objet du dixième du contenu des comptes rendus (9,9\%). Quant aux bibliographies et aux ouvrages de référence, qui constituent les outils de travail du bibliothécaire, ils ont retenu grandement l'attention des auteurs, soit $24,16 \%$ de l'ensemble. Notons de plus que la catégorie "autres ", avec ses 13,84\%, reçoit l'apport considérable des quinquennats 1955-1964 pendant lesquels les ouvrages recensés étaient fort disparates (volumes notamment de littérature, d'histoire, de religion); à partir de 1963, nous l'avons mentionné, le comité de rédaction ne retenait plus, pour comptes rendus, que les ouvrages intéressant notre discipline.

Le tableau 9 et le graphique 13 décrivent plus spécifiquement le matériel soumis pour recension. Où étaient publiés ces volumes et dans quelle

15. II y a un intérêt certain à souligner l'importance des contributions des présidents de l'ACBLF/ASTED : Edmond Desrochers (26 articles), Auguste-M. Morisset (19), Réal Bosa (17), Jean-Bernard Léveillé (14), Jean-Rémi Brault, Juliette Chabot et Henri Demers (13 articles chacun), C.Gabriel Allard, Paul Drouin et Pierre Matte ( 9 chacun), Patrick Allen, Germain Bélisle et Blanche Faucher ( 8 chacun), Claude Aubry, Maurice Auger, Georges-A Chartrand, Marcel Hudon et Joseph-R. Leduc ( 7 chacun), René Savard et Philippe Sauvageau (6 chacun), Marielle Durand (5), Claire Godbout (4).

16. Le Bulletin de l'ACBLF/Documentation et bibliothèques a publié 532 pages de comptes rendus, soit 34 en 1955-1959, 95,5 en $1960-1964,102,25$ en $1965-1969,131,75$ en $1970-$ 1974 et 168,5 en $1975-1979$. 
Tableau 8: Distribution des sujets des volumes recensés (comptes rendus)

\begin{tabular}{|c|c|c|c|c|c|c|}
\hline & $\begin{array}{c}1955-59 \\
\%\end{array}$ & $\begin{array}{c}1960-64 \\
\%\end{array}$ & $\begin{array}{c}1965-69 \\
\%\end{array}$ & $\begin{array}{c}1970-74 \\
\%\end{array}$ & $\begin{array}{c}1975-79 \\
\%\end{array}$ & $\begin{array}{c}\text { Total } \\
1955-79 \\
\%\end{array}$ \\
\hline \multicolumn{7}{|l|}{ Sujets } \\
\hline $\begin{array}{l}\text { 1. Fondements, théorie et philosophie de la } \\
\text { bibliothéconomie }\end{array}$ & 2,4 & 1,8 & 3,0 & - & 1,7 & 1,78 \\
\hline $\begin{array}{l}\text { 2. Formation (initiale et continue) du } \\
\text { bibliothécaire }\end{array}$ & 2,4 & 1,2 & - & - & 1,7 & 1,06 \\
\hline 3. Formation du technicien & - & - & - & 0,8 & - & 0,16 \\
\hline 4. Formation (autres) & - & - & - & 0,8 & - & 0,16 \\
\hline 5. Bibliothéconomie comparée et internationale & 2,4 & 0,6 & 1,0 & 0,8 & 2,5 & 1,46 \\
\hline 6. Censure & - & 1,8 & - & - & - & 0,36 \\
\hline 7. Histoire du livre et des bibliothèques & - & 1,8 & 8,0 & 2,4 & 3,3 & 3,1 \\
\hline 8. Édition et librairie & 2,4 & 0,6 & 4,0 & 1,6 & 3,3 & 2,38 \\
\hline 9. Administration des bibliothèques & - & 7,9 & 5,0 & 1,6 & 4,1 & 3,72 \\
\hline 10. Personnel & - & & - & - & - & - \\
\hline 11. Architecture et aménagement & 4,9 & 0,6 & - & 1,6 & 0,8 & 1,58 \\
\hline $\begin{array}{l}\text { 12. Services techniques (classification, } \\
\text { catalogage, indexation, acquisitions) }\end{array}$ & 14,7 & 4,9 & 12,0 & 10,5 & 7,4 & 9,9 \\
\hline $\begin{array}{l}\text { 13. Services publics (prêt, référence, formation } \\
\text { des usagers) }\end{array}$ & - & 5,5 & 1,0 & 0,8 & - & 1,46 \\
\hline 14. Collections (choix, coordination, élagage) & - & 1,2 & 1,0 & 2,4 & 3,3 & 1,58 \\
\hline 15. Sciences de l'information (sujets relatifs aux) & - & - & - & - & 0,8 & 0,16 \\
\hline $\begin{array}{l}\text { 16. Informatique et automatisation des } \\
\text { bibliothèques }\end{array}$ & - & & 2,0 & 3,2 & 1,7 & 1,38 \\
\hline 17. Banques et bases de données & - & - & - & - & 3,3 & 0,66 \\
\hline 18. Réseaux & - & - & & 0,8 & 2,5 & 0,66 \\
\hline 19. Documentation audiovisuelle & 2,4 & 0,6 & - & 2,4 & 5,0 & 2,08 \\
\hline 20. Publications officielles & 2,4 & - & 2,0 & 2,4 & 3,3 & 2,02 \\
\hline 21. Périodiques & 4,9 & - & 2,0 & 4,1 & 5,0 & 3,2 \\
\hline 22. Bibliographie (générale et spécialisée) & 14,7 & 7,9 & 13,0 & 20,2 & 19,8 & 15,12 \\
\hline 23. Ouvrages de référence & & 6,7 & 10,0 & 20,2 & 8,3 & 9,04 \\
\hline $\begin{array}{l}\text { 24. Livres et lecture des enfants et des } \\
\text { adolescents }\end{array}$ & 9,8 & 1,8 & 7,0 & 8,9 & 4,1 & 6,32 \\
\hline 25. Milieu et usagers & & - & - & 4,1 & 1,7 & 1.16 \\
\hline 26. Statistiques & 2,4 & 4,3 & 1,0 & - & - & 1,54 \\
\hline $\begin{array}{l}\text { 27. Associations de bibliothèques et de } \\
\text { bibliothècaires }\end{array}$ & - & - & - & - & - & 0 \\
\hline 28. L'État et les bibliothèques & & - & - & - & - & - \\
\hline 29. Archives & & - & 1,0 & - & 2,5 & 0,7 \\
\hline 30. Autres & 26,8 & 33,5 & 4,0 & 0,8 & 4,1 & 13,84 \\
\hline \multicolumn{7}{|l|}{ Genres de bibliothèques } \\
\hline 1. Nationale & - & 0,6 & 1,0 & 1,6 & - & 0,64 \\
\hline 2. Universitaire & & 1,2 & 5,0 & - & - & 1,24 \\
\hline 3. De collège & - & 4,3 & 1,0 & 0,8 & 2,5 & 1,72 \\
\hline $\begin{array}{l}\text { 4. Spécialisée et centre de documentation } \\
\text { (y compris bibliothèque gouvernementale) }\end{array}$ & - & - & 5,0 & 0,8 & & 1,16 \\
\hline 5. Scolaire & - & 4,9 & 3,0 & 1,6 & 5,0 & 2,9 \\
\hline 6. Publique & 7,3 & 6,1 & 8,0 & 4,8 & 2,5 & 5,74 \\
\hline
\end{tabular}


Tableau 9: Lieu d'édition des volumes recensés

\begin{tabular}{|l|c|c|c|c|c|}
\hline & $1955-59$ & $1960-64$ & $1965-69$ & $1970-74$ & $1975-79$ \\
$\%$ & $\%$ & $\%$ & $\%$ & $\%$ & $\%$ \\
\hline 1. - Québec & 21,0 & 42,9 & 40,2 & 60,2 & 71,0 \\
2. - Canada (à l'exception du Québec) & - & 11,3 & 24,1 & 15,0 & 9.8 \\
3. - France & 55,3 & 22,5 & 18,4 & 18,3 & 12,9 \\
4. Etats-Unis & 10,5 & 15,8 & 11,5 & 2,1 & 1,0 \\
5. - Grande-Bretagne & 2,6 & 3,7 & 3,4 & - & - \\
6. - Autres & 10,5 & 3,7 & 2,3 & 4,3 & 5,3 \\
\hline
\end{tabular}

langue? Ce qui ressort de ces données est la montée constante des publications québécoises au cours de la période, au détriment de l'apport français, américain et anglais. Avant 1960, à cause de la jeunesse et de la faiblesse de la discipline au Québec, les volumes venant de France constituaient la majorité des volumes recensés. A partir de 1970 , la production québécoise et canadienne en bibliothéconomie le permettant, et prenant acte que les ouvrages de langue anglaise dans notre discipline font l'objet de nombreux comptes rendus dans de non moins nombreuses revues, le comité de rédaction prenait la décision de ne retenir que les ouvrages de langue française, bilingues ou dans une autre langue, portant totalement ou partiellement sur le Québec. II ne faut donc pas se surprendre de la chute du pourcentage de volumes de langue anglaise publiés aux États-Unis et en Grande-Bretagne.

Comme nous l'avons souligné pour les articles, il nous apparaît intéressant de montrer l'évolution du nombre moyen de pages par compte rendu. Cette illustration permet de visualiser par la

\section{Graphique 13:}

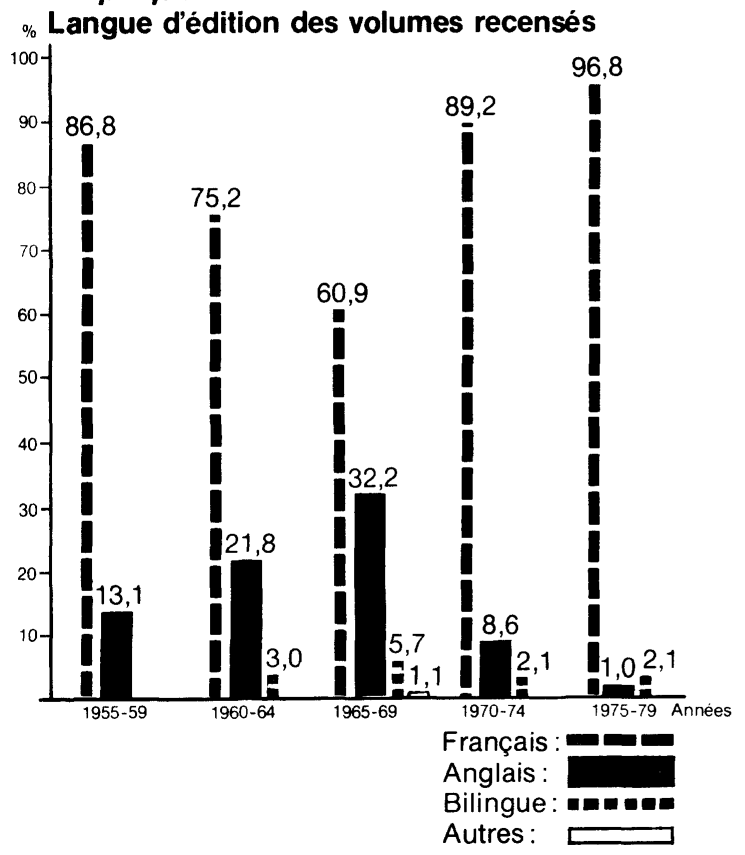

quantification la relative importance de l'espace réservé aux comptes rendus au cours des cinq lustres étudiés.

Le nombre moyen de pages n'a cessé d'augmenter depuis 1960. Nous croyons que le niveau atteint en 1975-1979 se stabilisera dans les années à venir et que cet espace est suffisant pour l'analyse et l'évaluation d'un ouvrage.

Le dernier tableau s'intéresse à la répartition des comptes rendus par auteurs durant la période 1955-1979. En construisant ce tableau, nous voulions vérifier si, à l'instar des articles, l'on pouvait constater une même ouverture de la revue à de nombreux collaborateurs.

\section{Tableau 10: Répartition comptes} rendus/auteurs (1955-1979)

\begin{tabular}{|cc|}
\hline $\begin{array}{c}\text { Nombre de } \\
\text { comptes rendus }\end{array}$ & Nombre d'auteurs \\
\hline 1 & 120 \\
2 & 30 \\
3 & 13 \\
4 & 4 \\
5 à 9 & 4 \\
10 à 14 & 5 \\
15 et plus & 4 \\
\hline
\end{tabular}

Le tableau 10 nous révèle que 120 auteurs différents n'ont écrit qu'un compte rendu; trente collaborateurs en ont rédigé deux et treize en ont fourni trois. A l'autre extrême, pendant les vingtcinq ans, seulement neuf personnes sont auteurs de dix comptes rendus et plus ${ }^{17}$. Ces auteurs ont fourni à eux -seuls $36,7 \%$ de l'ensemble des comptes rendus pendant les vingt-cinq ans alors que les auteurs de trois comptes rendus et moins constituent $48,5 \%$ de l'ensemble. Rappelons pour comparaison que les auteurs de dix articles et plus ne constituaient que $19,3 \%$ du total et que les auteurs de trois articles et moins représentaient

17. Parmi ces auteurs prolifiques, nous voyons Alvine Bélisle (10 comptes rendus), Paule Rolland-Thomas (11), Edmond Desrochers (12), Juliette Chabot (13), Jacques Paradis (14), Liana Van der Bellen (18), Monique Côté (21, tous produits entre 1960 et 1964), Laurent-G. Denis (26) et Georges-A. Chartrand (35 écrits entre 1955 et 1964). 
$50,2 \%$. La collaboration est similaire chez les auteurs de trois contributions et moins tant au plan des comptes rendus que des articles, mais il existe une différence notable dans l'apport des auteurs les plus prolifiques.

Notons enfin qu'il nous a été permis de constater un double phénomène : d'abord, le compte rendu s'avère dans de nombreux cas être un banc d'essai préalable à la collaboration plus substantielle qu'est un article; ensuite, un bon nombre d'auteurs ne signent que des comptes rendus.

\section{Conclusion}

L'examen et la dissection de ce périodique fondamental dans notre discipline nous ont permis de voir en lui à la fois un instrument et un reflet de l'effort ascendant de la maturation de la réflexion et de la pratique en bibliothéconomie québécoise au cours des années 1955-1979.

Notre analyse corrobore entièrement l'étude antérieure de Gilbert Gagnon, déjà citée, qui voyait pour la décennie 1950 la prédominance de l'humanisme chrétien en tant qu'idéologie dominante et la montée de l'idéologie scientifique au cours de la décennie 1960. L'auteur arrêtait son étude à l'année 1968. Pour nous, trois périodes se détachent dans l'évolution de ce périodique: la première, 1955-1963/1965, période dominée par le dévouement, l'apostolat chrétien, la nécessité de structuration d'une association pour laquelle le Bulletin est l'organe officiel ; la deuxième, 1963/ 1965-1972, au cours de laquelle on assiste à un effort de modernité, de normalisation continentale, à un dynamisme dans plusieurs genres de bibliothèques suite à une législation gouvernementale ou des transformations dans le monde de l'enseignement, à la formation universitaire des futurs praticiens, à l'introduction de nouvelles techniques, à la volonté de création d'un ordre professionnel, ce qui amène le Bulletin à approfondir ces diverses questions d'une manière empirique; et la troisième, depuis Documentation et bibliothèques, en 1973, voit la fin d'un monopole, à la suite de la création de la Corporation des bibliothécaires professionnels du Québec et de sa revue Argus ; émerge alors la volonté d'être le canal de la réflexion et de la recherche en information documentaire au Québec.

Nous avons discerné dans cette revue, à l'instar d'ailleurs de la documentation périodique spécialisée, des intérêts changeants, des modes intellectuelles, des mentalités professionnelles en mutation au fil des années et des périodes dans les sujets d'étude et dans leur approche.

La véritable recherche, avec ce qu'elle implique de cadre théorique, de méthodologie et de techniques de recherche, est jeune et précaire dans notre milieu. Mais les années présentes nous semblent particulièrement propices à sa stimulation et à son développement. Un périodique de recherche en information documentaire ne serait-il pas, à moyen terme, le véhicule nécessaire de ces travaux ? ${ }^{18}$

Par ailleurs, de plus en plus, il deviendra impératif à des spécialistes de l'information documentaire de scruter la production scientifique en général, et la leur en particulier, en amont comme en aval.
18. Voir - ou plutôt revoir - à ce sujet Une revue scientifique de bibliothéconomie au Québec. Rapport du comitè mixte ASTED/CBPQ sur les périodiques québécois en bibliothéconomie, Montréal, 1976, 67 p. 